EPJ Web of Conferences 66, 02030 (2014)

DOI: $10.1051 /$ epjconf/ 20146602030

(C) Owned by the authors, published by EDP Sciences, 2014

\title{
Precision mass measurements of short-lived nuclides for nu- clear structure studies at TITAN
}

\author{
A. Chaudhuri ${ }^{1, a}$, C. Andreoiu ${ }^{2}$, T. Brunner ${ }^{1,3, b}$, U. Chowdhury ${ }^{1,4}$, S. Ettenauer ${ }^{1,5, c}$, D. Frekers ${ }^{6}$, \\ A.T. Gallant ${ }^{1,5}$, A. Grossheim ${ }^{1}$, G. Gwinner ${ }^{4}$, R. Klawitter ${ }^{1}$, A.A. Kwiatkowski ${ }^{1}$, K.G. Leach ${ }^{1}$, \\ A. Lennarz ${ }^{6}$, D. Lunney ${ }^{7}$, T. D. Macdonald ${ }^{1,5}$, B.E. Schultz ${ }^{1}$, S. Seeraji ${ }^{2}$, M.C. Simon ${ }^{1}$, V.V. \\ Simon $^{1,8, d}$, and J. Dilling ${ }^{1,5}$ \\ ${ }^{1}$ TRIUMF, 4004 Wesbrook Mall, Vancouver, BC, V6T 2A3, Canada \\ ${ }^{2}$ Department of Chemistry, Simon Fraser University, Burnaby, BC V5A 1S6, Canada \\ ${ }^{3}$ Physik Department E12, Technische Universität München, 85748 Garching, Germany \\ ${ }^{4}$ Department of Physics and Astronomy, University of Manitoba, Winnipeg, MB, R3T 2N2, Canada \\ ${ }^{5}$ Department of Physics and Astronomy, University of British Columbia, Vancouver, BC, V6T 1Z1, Canada \\ ${ }^{6}$ Institut für Kernphysik, Westfälische Wilhelms-Universität, 48149 Münster, Germany \\ ${ }^{7}$ CSNSM-IN2P3-CNRS, Université de Paris Sud, 91405 Orsay, France \\ ${ }^{8}$ Max-Planck-Institut für Kernphysik, Saupfercheckweg 1, 69117 Heidelberg, Germany
}

\begin{abstract}
TITAN (TRIUMF's Ion Trap for Atomic and Nuclear science) at TRIUMF's rare isotope beam facility ISAC is an advanced Penning trap based mass spectrometer dedicated to precise and accurate mass determinations. An overview of TITAN, the measurement technique and a highlight of recent mass measurements of the short-lived nuclides important to the nuclear structure program at TITAN are presented.
\end{abstract}

\section{Introduction}

Accurate and precise mass values of atomic nuclides are important for many disciplines in physics including the study of nuclear structure [1], the study of the production of elements in stellar nucleosynthesis [2], and the test of fundamental symmetries, such as the conserved vector current (CVC) hypothesis in super allowed $\beta$ decays [3]. However, the required relative uncertainty of the mass depends on the physics under investigation and generally varies from $\delta m / m=10^{-6}$ to $10^{-11}$ [4]. Today Penning traps are widely accepted as the most accurate tool for high-precision mass spectrometry of atomic nuclides [5]. The coupling of Penning traps to rare-isotope-beam facilities has revolutionized the field of mass spectrometry of exotic nuclides. Presently, the operational Penning traps for mass measurements of exotic nuclides are ISOLTRAP [6], CPT [7], JYFLTRAP [8], SHIPTRAP [9], LEBIT [10], TRIGA-SPEC [11], and TITAN [12].

The TITAN Penning trap mass spectrometer is coupled to the rare-isotope-beam facility ISAC [13]

\footnotetext{
a e-mail: ankur@triumf.ca

${ }^{\mathrm{b}}$ Present address: Department of Physics, Stanford University, Stanford, CA 94305, USA

${ }^{\mathrm{c}}$ Present address: Department of Physics, Harvard University, Cambridge, MA 02138, USA

$\mathrm{d}$ Present address: Helmholtz-Institut Mainz, 55128 Mainz, Germany
}

This is an Open Access article distributed under the terms of the Creative Commons Attribution License 2.0, which permits unrestricted use, distribution, and reproduction in any medium, provided the original work is properly cited. 


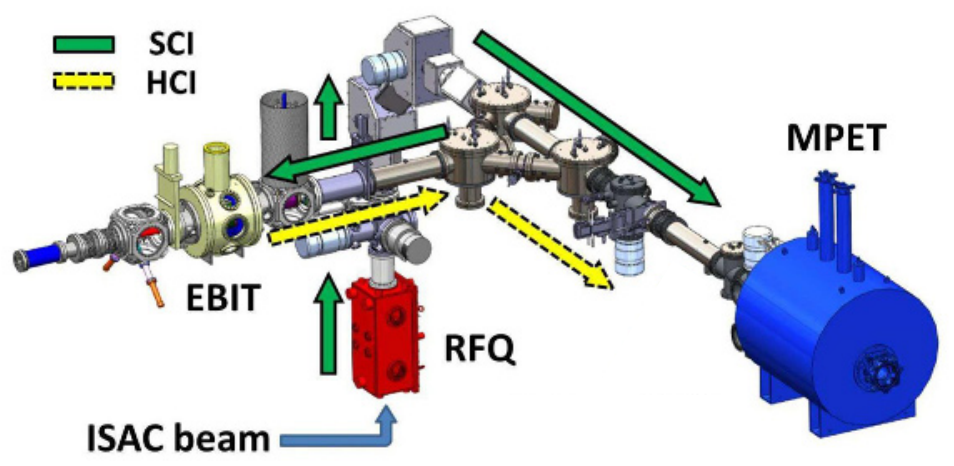

Figure 1. TITAN experimental setup and the ion-beam direction of singly-charged ions (SCI) and highly-charged ions (HCI).

at TRIUMF and provides unique capabilities for precision mass measurement of short-lived nuclides as was demonstrated by the measurement of ${ }^{11} \mathrm{Li}\left(t_{1 / 2}=8.8 \mathrm{~ms}\right)[14,15]$. Another unique feature of the TITAN experiment is the use of highly-charged short-lived ions, which provides an improvement in experimental precision for the mass measurement $[16,17]$. During the recent years TITAN has provided accurate and precise mass values of atomic nuclides to study nuclear structure [14, 18-25], nuclear astrophysics [26], the weak interaction [17], and neutrino physics [27].

\section{TITAN experimental setup for precision mass measurements}

The short-lived ions delivered from ISAC [13] or the stable ions delivered from an off-line ion source are transported to the helium gas-filled radio-frequency quadrupole (RFQ) cooler and buncher [28] (see figure 1). The ions are cooled, accumulated and then extracted as a low-emittance bunched beam. The ion-bunch is sent either directly to the measurement Penning trap (MPET) for mass measurement using singly-charged ions, or deflected towards the EBIT [29] for charge breeding and consequently sent to the MPET for mass measurement using highly-charged ions. A Bradbury-Nielsen gate [30] for selecting a specific mass-to-charge ratio prior to the injection into the MPET is employed. The cyclotron frequency of the trapped ion in the Penning trap is measured by a time-of-flight ion-cyclotron-resonance (TOF-ICR) detection method [31]. The ion-cyclotron frequency is obtained from the individual TOF resonance. Figure 2 shows an example of the TOF resonance of short-lived ${ }^{31} \mathrm{Mg}^{+1}$ ion. The mass of an ion can be determined by measuring the ion cyclotron frequency

$$
v_{c}=\frac{1}{2 \pi}\left(\frac{q}{m_{\text {ion }}}\right) B
$$

of the ion trapped in the Penning trap, where $B$ is the magnetic field strength of the Penning trap, and $q / m_{\text {ion }}$ is the charge-to-mass ratio of the ion. 


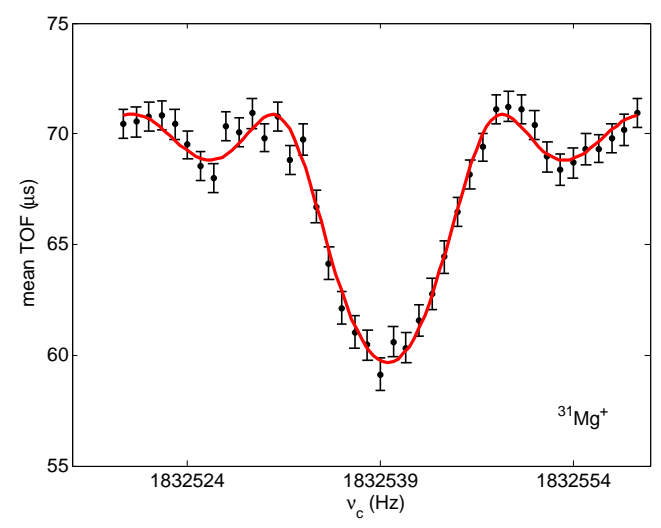

Figure 2. Average time-of-flight of singly-charged ${ }^{31} \mathrm{Mg}^{+}$ions as a function of the excitation frequency applied in the precision Penning trap for an excitation time of $97 \mathrm{~ms}$. The solid line represents a fit of the expected resonance curve to the data points [31].

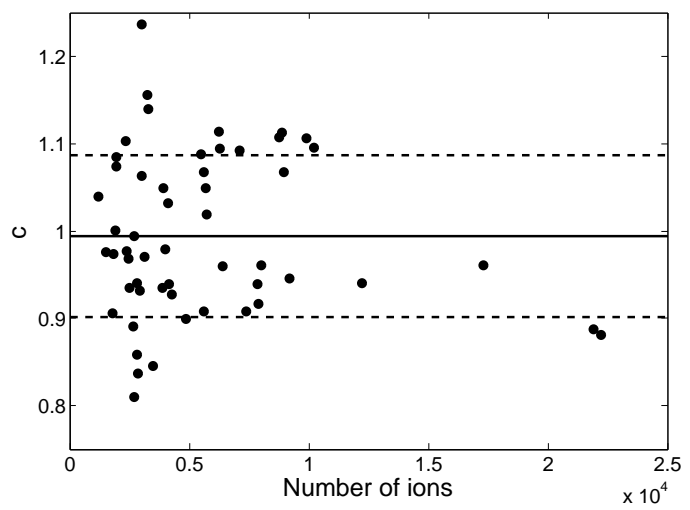

Figure 3. The empirical factor $c$ as a function of the number of ions $(n)$ detected from different ion-cyclotron-frequency measurements of ${ }^{16} \mathrm{O}_{2}{ }^{+1}$ ions. The solid line indicates the average value of $c$, and the dashed lines indicate its one-standard-deviation uncertainty.

For a TOF resonance, the relative statistical uncertainty of the experimentally determined cyclotron frequency can be described by the following relation [32]:

$$
\frac{\delta v_{c}}{v_{c}}=\frac{c}{v_{c} T_{R F} \sqrt{n}} .
$$

Here, $n$ is the total number of detected ions, $T_{R F}$ is the duration of the quadrupole excitation in the Penning trap, $v_{c}$ is the cyclotron frequency and $c$ is an empirical factor depending on specifics of the trap, extraction path, and measurement settings. According to equations (1) and (2), the precision of the Penning trap mass spectrometer increases with increasing quadrupole excitation time, number of ions recorded in a resonance, magnetic field strength, and charge state of the ion. Employing the EBIT charge-breeder, TITAN is capable of boosting the measurement precision by measuring highlycharged ions. The number of recorded ions $n$ is limited by the production yield of exotic ions and the available experimental time, and the applied quadrupolar excitation time $T_{R F}$ is limited by the half-life of the isotope. During a recent investigation the parameter $c$ of equation (2) was determined by the cyclotron-frequency measurements of singly-charged ${ }^{16} \mathrm{O}_{2}{ }^{+1}$ ions. The $T_{R F}$ was chosen to be between $18 \mathrm{~ms}$ and $997 \mathrm{~ms}$. The result of these measurements is shown in figure 3 . The average value 
of $c$ from this investigation was found to be 0.99(9), which is comparable to the value of $c=0.898(8)$ obtained from a series of cyclotron frequency measurements of singly-charged ${ }^{85} \mathrm{Rb}$ ions conducted at ISOLTRAP [33].

\section{Recent measurements for nuclear structure studies}

The nuclear structure program at TITAN has focussed on three distinct areas of the nuclear chart (figure 4): neutron-rich light nuclides, neutron-rich $\mathrm{Ca}$ and $\mathrm{K}$ nuclides, and the island of inversion nuclides around ${ }^{32} \mathrm{Mg}$. The related mass measurements of short-lived nuclides conducted at TITAN are summarized below.

\subsection{Light neutron-rich nuclides (neutron-halos)}

The properties of light halo nuclides provide stringent tests for nuclear models. High-precision mass measurements of such nuclides serve as valuable benchmarks for nuclear structure theory as the mass is relevant for two independent parameters, the two-neutron separation energy $\left(\mathrm{S}_{2 n}\right)$ and the charge radius. TITAN was able to provide accurate and precise mass values for light nuclear system including one-neutron halo nuclides ${ }^{11,12} \mathrm{Be}$, two-neutron halo nuclides ${ }^{6} \mathrm{He}$ and ${ }^{11} \mathrm{Li}$, and the four-neutron halo nuclide ${ }^{8} \mathrm{He}$. Note that ${ }^{12} \mathrm{Be}$ is not a halo in ground state, but its long-lived $0_{2}^{+}$state shows a neutron halo-like structure [34]. The mass values of ${ }^{6,8} \mathrm{He}[18,19]$ and ${ }^{11} \mathrm{Li}[14]$ were critically relevant for the determination of the halo charge-radius of the respective nuclides [35-37]. The first direct mass measurement of ${ }^{6} \mathrm{He}$ along with a precise mass value for ${ }^{8} \mathrm{He}$ provided stringent tests for three-body forces in an extreme neutron-rich system [19]. TITAN mass measurements of ${ }^{9-11} \mathrm{Be}$ removed atomic mass uncertainties as significant sources of error in determining the relative nuclear charge radius from the isotope shift [20]. The mass measurement of ${ }^{12} \mathrm{Be}$ [21] performed at TITAN contributed to the charge radius determination of ${ }^{12} \mathrm{Be}[38]$, and to a test of isobaric multiplet mass equation (IMME) [39].

\subsection{Neutron-rich $\mathrm{K}$ and Ca nuclides}

Direct mass measurements of neutron-rich ${ }^{44,47-51} \mathrm{~K}$ and ${ }^{49-52} \mathrm{Ca}$ isotopes [24, 25] have been carried out with the TITAN Penning trap to gain insight into a predicted shell closure around $N=34$. The newly measured ${ }^{49} \mathrm{~K}$ mass value increases the neutron-shell gap energy at $N=28$ by approximately $1 \mathrm{MeV}$ [24] from the shell gap energy calculated from the mass values in previous atomic mass evaluation [40]. Also, the masses of ${ }^{51,52} \mathrm{Ca}$ show an increase in binding energy [25]. The mass of

${ }^{52} \mathrm{Ca}$ was found to be more bound by $1.74 \mathrm{MeV}$ compared to [40]. Recent calculation based on threenucleon $(3 \mathrm{~N})$ forces [41] predicted an increased binding around $N=32$. The two-neutron separation energies $\left(S_{2 n}\right)$ obtained from the TITAN mass measurements remarkably agree with the $S_{2 n}$ predicted from the improved theoretical calculation that include $3 \mathrm{~N}$ forces. This emphasizes the importance of accurate and precise Penning trap mass measurements to test the predictive power of theory, which was also confirmed by multi-reflection TOF mass measurements by ISOLTRAP [42].

\subsection{Island of inversion nuclides}

The disappearance of the traditional neutron magic number $N=20$, a case of shell quenching, remains at the center of experimental and theoretical nuclear physics. In a recent experiment at TITAN, highprecision mass measurements of short-lived ${ }^{30-34} \mathrm{Mg},{ }^{29-31} \mathrm{Na}$ and ${ }^{29-34} \mathrm{Al}$ nuclides around $N=20$ 


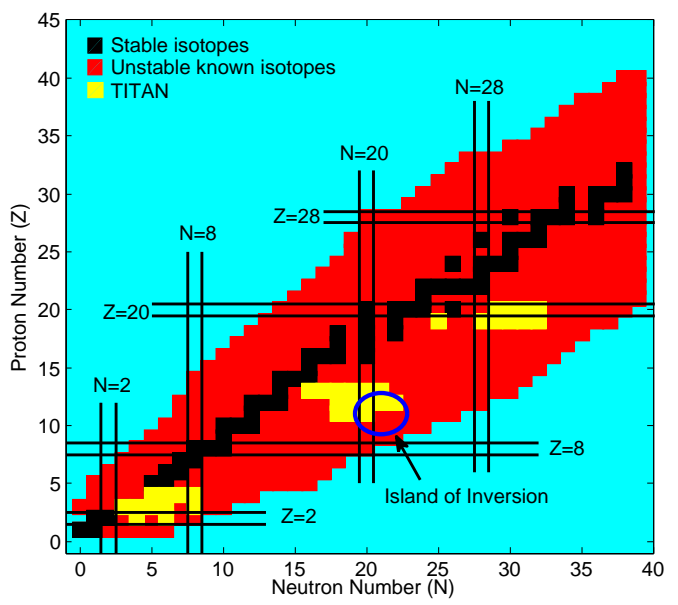

Figure 4. Nuclides measured at TITAN for the nuclear structure studies (yellow rectangle) and the position of neutron $(N)$ and proton $(Z)$ magic numbers (solid lines) in the nuclear landscape. The stable and unstable isotopes are shown in black and red rectangles, respectively.

have been carried out. Among all on-line Penning trap mass spectrometers currently in operation, TITAN is the only facility capable of performing such high-precision mass measurements on very short half-lived isotopes. The new mass measurements of island of inversion nuclides ${ }^{32,34} \mathrm{Mg}$ and ${ }^{31} \mathrm{Na}$ provided direct evidence of the extinction of $N=20$ neutron-shell closure around $Z=12$ and a break-down of the established nuclear shell model in this region from ground state properties [43].

\section{Acknowledgements}

This work has been supported by the Natural Sciences and Engineering Research Council (NSERC) of Canada and the National Research Council (NRC) of Canada. A.T.G. acknowledges support from the NSERC CGS-D program, A.L. from the Deutsche Forschungsgemeinschaft (DFG) under grant no. FR 601/3-1, T.D.M. from the NSERC CGS-M program. We thank M. Good for providing technical support for the TITAN experiment.

\section{References}

[1] D. Lunney et al., Rev. Mod. Phys. 75, 1021 (2003)

[2] H. Schatz, Int. J. Mass Spectrom. 251, 293 (2006)

[3] J. C. Hardy and I. S. Towner, Phys. Rev. C 71, 055501 (2005)

[4] K. Blaum, Phys. Rep. 425, 1 (2006)

[5] K. Blaum, J. Dilling and W. Nörtershäuser, Phys. Scr. T152, 014017 (2013)

[6] M. Mukherjee et al., Eur. Phys. J. A 35, 1 (2008)

[7] G. Savard et al., Int. J. Mass Spectrom. 251, 252 (2006)

[8] V.S. Kolhinen et al., Nucl. Instrum. Methods A 528, 776 (2004)

[9] M. Block et al., Eur. Phys. J. D 45, 39 (2007)

[10] R. Ringle et al., Nucl. Instrum. Methods A 604, 536 (2009)

[11] J. Ketelaer et al., Nucl. Instrum. Methods A 594, 162 (2008)

[12] J. Dilling et al., Int. J. Mass Spectrom. 251, 198 (2006)

[13] M. Dombsky et al., Rev. Sci. Instrum. 71, 978 (2000)

[14] M. Smith et al., Phys. Rev. Lett. 101, 202501 (2008)

[15] A. Chaudhuri et al., submitted to Appl. Phys. B 
[16] S. Ettenauer et al., Int. J. Mass Spectrom., in press (http://dx.doi.org/10.1016/j.ijms.2013.04.021)

[17] S. Ettenauer et al., Phys. Rev. Lett. 107, 272501 (2011)

[18] V. L. Ryjkov et al., Phys. Rev. Lett. 101, 012501 (2008)

[19] M. Brodeur et al., Phys. Rev. Lett. 108, 052504 (2012)

[20] R. Ringle et al., Phys. Lett. B 675, 170 (2009)

[21] S. Ettenauer et al., Phys. Rev. C 81, 024314 (2010)

[22] M. Brodeur et al., Phys. Rev. Lett. 108, 212501 (2012)

[23] M. Brodeur et al., Phys. Rev. C 80, 044318 (2009)

[24] A. Lapierre et al., Phys. Rev. C 85, 024317 (2012)

[25] A.T. Gallant et al., Phys. Rev. Lett. 109, 032506 (2012)

[26] V.V. Simon et al., Phys. Rev. C 85, 064308 (2012)

[27] D. Frekers et al., Phys. Lett. B 722, 233 (2013)

[28] T. Brunner et al., Nucl. Instrum. Methods A 676, 32 (2012)

[29] A. Lapierre et al., Nucl. Instrum. Methods A 624, 54 (2010)

[30] T. Brunner et al., Int. J. Mass Spectrom. 309, 97 (2012)

[31] M. König et al., Int. J. Mass Spectrom. 142, 95 (1995)

[32] G. Bollen, Nucl. Phys. A 693, 3 (2001)

[33] A. Kellerbauer et al., Eur. Phys. J. D 22, 53 (2003)

[34] R. Kanungo et al., Phys. Lett. B 682, 391 (2010)

[35] L.B. Wang et al., Phys. Rev. Lett. 93, 142501 (2004)

[36] P. Mueller et al., Phys. Rev. Lett. 99, 252501 (2007)

[37] R. Sanchez et al., Phys. Rev. Lett. 96, 033002 (2006)

[38] A. Krieger et al., Phys. Rev. Lett. 108, 142501 (2012)

[39] M.F. Jager et al., Phys. Rev. C 86, 011304(R) (2012)

[40] G. Audi, A. H. Wapstra, C. Thibault, Nucl. Phys. A 729, 337 (2003)

[41] J.D. Holt, T. Otsuka, A. Schwenk, and T. Suzuki, J. Phys. G 39, 085111 (2012)

[42] F. Wienholtz et al., Nature 498, 346 (2013)

[43] A. Chaudhuri et al., in preparation 